\title{
Evaluating the inflammatory activity in Crohn's disease using magnetic resonance diffusion kurtosis imaging
}

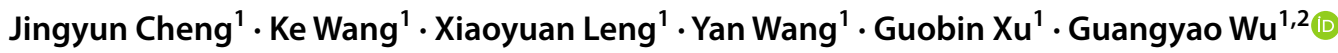

Published online: 15 May 2019

(c) The Author(s) 2019

\begin{abstract}
Objectives To explore the feasibility of diffusion kurtosis imaging (DKI) for evaluating inflammatory activity in Crohn's disease $(\mathrm{CD})$.

Materials and methods In all, $51 \mathrm{CD}$ patients were included, who were performed with consecutive enteroscopy, MR and DKI ( $b$ values $=0-2000 \mathrm{~mm}^{2} / \mathrm{s}$ ). The lesions of bowel segments were graded as inactive (0-2), mild (3-6), and moderatesevere group $(>6)$ based on simplified endoscopic activity score for Crohn's disease (SES-CD), The abilities of the parameters of DKI and DWI in grading different activity lesions were compared.

Results One hundred and twenty-seven bowel segments including inactive (15), mild (45) and moderate-severe (67) were analyzed. ADC $(r=-0.627, p<0.001), D_{\text {app }}(r=-0.381, p<0.001)$ and $K_{\text {app }}(r=0.641, p<0.001)$ were correlated with SES-CD. These parameters were significantly different among the three groups (all $p<0.001$ ). ROC analysis found $\mathrm{ADC}$ had the highest accuracy ( $\mathrm{AUC}=0.884, p<0.001$ ) to differentiate inactive from active group with the threshold at $0.865 \times 10^{-3} \mathrm{~mm}^{2} / \mathrm{s}$, which was slightly higher than $K_{\text {app }}(\mathrm{AUC}=0.867, p<0.001)$ with the threshold at 0.645 , and was obviously higher than $D_{\text {app }}(\mathrm{AUC}=0.726, p=0.005)$. Similarly, ADC also had the highest accuracy $(\mathrm{AUC}=0.846, p<0.001)$ to differentiate inactive-mild from moderate-severe group with the threshold at $0.825 \times 10^{-3} \mathrm{~mm}^{2} / \mathrm{s}$, and minimally higher than $K_{\text {app }}\left(\mathrm{AUC}=0.843, p<0.001\right.$ ) with the threshold at 0.695 , and obviously higher than $D_{\text {app }}(\mathrm{AUC}=0.690, p<0.001)$. Conclusion DKI is feasible and comparable to conventional DWI for the evaluation of inflammatory activity in CD.
\end{abstract}

Keywords Crohn's disease $\cdot$ Diffusion kurtosis $\cdot$ Imaging diffusion $\cdot$ Weighted imaging $\cdot$ Simplified endoscopic activity score for Crohn's disease

Guobin Xu

guobinxu@126.com

$\triangle$ Guangyao Wu

wuguangy2002@163.com

Jingyun Cheng

chengjy2017@126.com

Ke Wang

wangke2013@whu.edu.cn

Xiaoyuan Leng

lengxy18@163.com

Yan Wang

1749036341@qq.com

1 Department of Radiology, Zhongnan Hospital of Wuhan University, Wuhan 430071, Hubei, People's Republic of China

2 Radiology Department, Shenzhen University General Hospital and Shenzhen University Clinical Medical Academy, Shenzhen, People's Republic of China

\section{Introduction}

Crohn's disease (CD) is a chronic relapsing and remitting disorder that can involve entire gastrointestinal tract and cause irreversible intestinal damage [1]. Accurate assessment of disease activity is critical for therapeutic decision, clinical management, and follow-up of CD patients [1-4]. Magnetic resonance enterography (MRE) is increasingly used for diagnosis and assessment of CD benefitting from its non-ionizing radiation and excellent soft tissue contrast. And several studies have demonstrated that the magnetic resonance index of activity (MaRIA) is well correlated with Crohn's Disease Endoscopic Index of Severity (CDEIS) or SES-CD $[2,5,6]$ and widely used for monitoring therapeutic response [2-4].

Diffusion weighted imaging (DWI), as a method of the only non-invasive assessment of free diffusion of water molecules, has been increasingly applied to evaluate pediatric 
patient or renal failure patients. Several studies found that DWI is noninferiority to contrast-enhanced MR imaging for the evaluation of inflammation and diagnosis of complications in $\mathrm{CD}[7,8]$. And apparent diffusion coefficient (ADC), the quantitative parameter of DWI, has been increasingly used for evaluating disease activity in $\mathrm{CD}[8,9]$. However, conventional ADC was based on assumption that the displacement of water molecular was an ideal Gaussian distribution without any restriction. Actually, water molecular diffusion in complex biological tissue tends to deviate from a Gaussian distribution, owing to cellular microstructural barriers.

Diffusion kurtosis imaging is an advanced DWI model that quantifies non-Gaussian behavior of diffusion, yielding additional parameters, apparent kurtosis coefficient $\left(K_{\text {app }}\right)$, reflecting the deviation degree from the ideal Gaussian curve, and the corrected ADC value, which quantifies the non-Gaussian behavior of water molecular diffusion [10]. Recent years, DKI has been widely applied to the preoperative diagnosis, grading and postoperative surveillance of cancer [11-13]. Nevertheless, few studies applied DKI to evaluate the activity of autoimmune inflammation diseases, especially in the inflammatory bowel disease. Hence, the objectives of this study are to explore the feasibility of DKI for the evaluation of inflammatory activity in Crohn's disease $(\mathrm{CD})$.

\section{Materials and methods}

\section{Patients}

This is a retrospectively observational study conducted from July 2016 to September 2018, the patients consecutively performed MRE with DKI sequence and enteroscopy at our hospital were selected. Informed consent was obtained from all patients. Inclusion criteria were the following: (a) the diagnosis of CD was established; (b) the interval between MR examination and enteroscopy was within 14 days; (c) intestinal dilatation was good, and it did not affect the assessment. Exclusion criteria were the following: (a) the diagnosis of $\mathrm{CD}$ is not confirmed; (b) intestinal dilatation was poor and can impact assessment; (c) the interval between MR examination and endoscopy exceeds 2 weeks; (d) bowel resection before MR examination or enteroscopy; (e) patients had contraindications for MR examinations. Clinical disease activity was assessed using the Harvey-Bradshaw Index (HBI); C-reactive protein (CRP) and erythrocyte sedimentation rate (ESR) were examined between the two examinations.

Sixty-seven patients were originally considered in our database. Besides, ten patients were excluded because of bowel resection before the two examinations; one patient was ulcerative colitis, one patient was excluded due to the interval between the two examinations exceeded 14 days; four patients were intestinal tuberculosis. Finally, 51 patients (37 males and 14 females) were included in the study.

\section{MRE protocol}

Patients were required to take polyethylene glycol electrolyte (PGE) solution for bowel cleaning the night before the procedure, and to take oral $1500-2000 \mathrm{ml}$ of $2.5 \%$ mannitol solution (depending on patient's physique and comfort) $1 \mathrm{~h}$ before the procedure. A $10 \mathrm{mg}$ of anisodamine was slowly injected intramuscularly into the buttocks to induce gastrointestinal hypotonia $10 \mathrm{~min}$ before MRE.

The MRI examinations were performed on a 3.0 T MR scanner (Magnetom Prisma, Siemens Healthcare, Erlangen, Germany) with two dedicated 32-channel abdominal phased-array coil. Patients were in the supine position. Coronal and axial fat-saturated T2-weighted half-Fourier single shot turbo spin-echo (HASTE), coronal HASTE were taken. Coronal T1-weighted volumetric interpolated breath-hold examination (VIBE) sequences were also obtained at baseline and then $30,60,90,180 \mathrm{~s}$ after injection of $0.2 \mathrm{ml} / \mathrm{kg}$ body weight $10 \mathrm{ml}$ (10 ml: $4.69 \mathrm{~g}$ ) gadopentetate dimeglumine (Magnevist; Beijing Beilu Pharmaceutical Co., Ltd). DKI was performed by axial free-breathing using a single shot spin-echo echo planar imaging (SE-EPI) sequence in three orthogonal directions with five $b$ values $(200,500$, $1000,1500,2000 \mathrm{~s} / \mathrm{mm}^{2}$ ) before contrast agent administration. The total acquisition time of DKI was 4 min $32 \mathrm{~s}$. MR protocol and sequence parameters were summarized in Table 1.

\section{Enteroscopy examination and evaluation}

Patients followed a bowel cleansing protocol via oral ingestion of $2000 \mathrm{ml}$ of polyethylene glycol (PEG, Fortrans, BEAUFOUR ISPEN, Tianjin) on the evening before examination and $2000 \mathrm{ml}$ the morning of examination. Enteroscopy was performed under anesthesia with propofol (Fresenius Kabi Austria GmbH, Austria). All enteroscopies (oral-into the jejunum about $350 \mathrm{~cm}$ away from the pylorus and the anal-into the ileum about $150 \mathrm{~cm}$ away from the ileocecal valve) were performed by two experienced endoscopists (L.J. and C.M.) with single-balloon enteroscopy (SIF-Q260; Olympus, Tokyo, Japan). The severity and extent of enteroscopic lesions were retrospectively assessed by an experienced endoscopist (L.Q.) without knowledge of MRE results according to the simplified endoscopic score for Crohn's disease (SES-CD) [14]. The SES-CD was applied to each segment (jejunum, proximal ileum, terminal ileum, cecum/right colon, transverse colon, left/sigmoid colon and rectum) to obtain a segmental SES$\mathrm{CD}$. The terminal ileum was defined as the segment $\leq 10 \mathrm{~cm}$ 
Table 1 Scanning parameters of magnetic resonance enterography protocol and DKI

\begin{tabular}{lllll}
\hline Sequence & T2WI-HASTE & T2WI-HASTE & DKI & $\begin{array}{l}\text { Post-contrast (0, 30, } \\
60,90,180 \mathrm{~s}) \text { VIBE }\end{array}$ \\
\hline Plane & Axial & Coronal & Axial & Coronal \\
No. of slices & 35 & 35 & 50 & 112 \\
Repetition time (ms) & 1000 & 1100 & 7400 & 4.21 \\
Echo time (ms) & 83 & 92 & 58 & 1.34 \\
PAT & 4 & 3 & 2 & 6 \\
Section thickness $(\mathrm{mm})$ & 5.0 & 4.0 & 6.0 & 1.5 \\
Field of view $(\mathrm{mm})$ & $340 \times 289$ & $400 \times 400$ & $330 \times 300$ & $450 \times 366$ \\
Voxel size & $1.1 \times 1.1 \times 5.0$ & $1.3 \times 1.3 \times 4.0$ & $1.3 \times 1.3 \times 6.0$ & $1.4 \times 1.4 \times 1.5$ \\
Flip angle $\left({ }^{\circ}\right)$ & 150 & 150 & 90 & 10 \\
Fat saturation & $\mathrm{N}$ & Y/N & $\mathrm{N}$ & $\mathrm{Y}$ \\
$b$ values $\left(\mathrm{s} / \mathrm{mm}^{2}\right)$ & & & $200,500,1000,1500,2000$ \\
\hline
\end{tabular}

VIBE volumetric interpolated breath-hold examination, $P A T$ parallel acquisition technique from the ileocecal valve. Proximal ileum was defined as the segment from $>10 \mathrm{~cm}$ from the ileocecal valve to the borderline of between jejunum and ileum [4]. Each segment was graded as inactive (0-2), mild (3-6), or moderate-severe $(\geq 7)$ by SES-CD. Inactive segments included normal segments (intestinal mucosa was absolutely normal) and some segments with lesions (SES-CD $\leq 2$ ). Normal segments were not scored because the purpose of the study was to retrospectively assess the lesion activity. There were 213 normal segments in the study. Finally, there are 28 inactive, 49 mild and 67 moderate-severe.

\section{Imaging analysis}

Two radiologists, one junior (with 3 years of experience in abdominal imaging and MRE, C.J.) and one senior (with more than 15 years of experience in abdominal imaging and MRE, X.G.) independently reviewed the anonymized MRE examinations on a picture-archiving and communication system (PACS) viewing station (Carestream Health Inc, Toronto, Canada). In case of discrepancies, consensus was reached after discussion between the two radiologists. For each segmental analysis, the same division as enteroscopy was applied. MRE variables were evaluated in each segment including bowel wall thickening (bowel wall thickness $>3 \mathrm{~mm}$ in good intestinal dilatation), presence of ulcer (deep depression in the mucosal surface of thickened bowel segment), presence of mucosal edema (T2 hyperintensity relative to psoas muscle) and relative contrast enhancement (RCE), which was calculated by $\mathrm{RCE}=\left[\left(\mathrm{SI}_{\text {post }}-\mathrm{SI}_{\mathrm{pre}}\right)\right.$ / $\left.\left(\mathrm{SI}_{\text {pre }}\right)\right] \times 100 \times\left(\mathrm{SD}\right.$ noise pre $/ \mathrm{SD}$ noise $\left.{ }_{\text {post }}\right)$, where $\mathrm{SI}_{\text {pre }}$ and $\mathrm{SI}_{\text {post }}$ denote wall signal intensity, SD noise pre $_{\text {and }} \mathrm{SD}$ noise $_{\text {post }}$ denote standard deviations of noise outside of the body before and after gadolinium injection. $\mathrm{SI}_{\text {post }}$ and SD noise $_{\text {post }}$ were measured on the 3D VIBE images at $70 \mathrm{~s}$ after contrast enhancement. Three adjacent circular region of interest (ROI) were drawn in each selected segment of abnormal bowels. In case of dominantly intense mucosal enhancement, the ROIs were placed as close as possible into the inner mucosal surface. The mean value of three ROIs was calculated for each segment. Each ROI had an area between 3 and $6 \mathrm{~mm}^{2}$ [15]. To measure the background noise (standard deviation), a single ROI between 60 and $80 \mathrm{~mm}^{2}$ was placed outside the abdomen on the same field of view [15]. The MaRIA was calculated by [6]: MaRIA $=1.5 \times$ wall thickness $+0.02 \times \mathrm{RCE}+5 \times$ edema $+10 \times$ ulcers.

DKI and DWI data were analyzed using an in-house program written in MATLAB (version R2013b, MathWorks, MA, USA). The DKI parameter was fitted according to the following equation [10-13]:

$\frac{S_{\mathrm{b}}}{S_{0}}=\exp \left[-\mathrm{b} \times D_{\mathrm{app}}+\frac{1}{6} \times b^{2} \times\left(D_{\mathrm{app}}\right)^{2} \times K_{\mathrm{app}}\right]$,

where $S_{0}$ is signal intensity when $b$ value is $0, S_{\mathrm{b}}$ is the signal intensity for a given $b$ value $\left(\mathrm{s} / \mathrm{mm}^{2}\right)$, representing the diffusion gradient. $D_{\text {app }}$ is the ADC corrected by the nonGaussian model, representing true diffusivity. $K_{\text {app }}$ is kurtosis, indicating the extent of water molecule diffusion that deviates from the Gaussian distribution.

The ADC is the mean value obtained using all $b$ values that were fitted with a conventional mono-exponential model according to the following equation $[11,12]$ :

$S_{b}=S_{0} \times \exp (-b \times \mathrm{ADC})$,

where $S_{0}$ is signal intensity when b value is $0, S_{\mathrm{b}}$ is the signal intensity for a given $b$ value, and ADC is the apparent diffusion coefficient.

Two radiologists (W.K. with 6 years of experience in abdominal imaging and MRE and W.G. with more than 15 years of experience in abdominal imaging and MRE) reviewed MRE images, who were blind to clinical and enteroscopy results. Because of low resolution of DKI data, the 
target segments were selected by location-by-location based on MRE. Although we spared our effort to evaluate the lesions of each segments by location-by-location between endoscopy and MRE, there were still some inactive (SES$\mathrm{CD} \leq 2$ ) lesions not detected on MRE, however, which were actually detected on endoscopy, because MRE was hard to display minimal lesions, such as erosion, erythema, edema, small ulcers etc. Finally, compared with enteroscopy results, there were 13 inactive and four mild segments not detected by MRE, however, all moderate-severe segments were detected. The lesions displayed by MR were all detected by enteroscopy. So we selected the lesions that were detected by both endoscopy and MRE. Each ROI was manually drawn along the border of the brightest signal region of bowel wall on three consecutive slices (the slice with the prominent lesion section and the adjacent up and down slices) [16]. Then, all the parameters, including mean ADC, mean $D_{\text {app}}$, and $K_{\text {app }}$, were calculated by voxel using the whole volume method.

\section{Statistical analysis}

Statistical analysis was performed using the IBM SPSS Statistics for Windows Version 25.0 (IBM Corp., Armonk, NY). The tests were two-sided, with a Type I error set at $\alpha=0.05$. Categorical variables were presented as frequency and percentages. Continuous variables were tested by the Kolmogorov-Smirnov test and the Levene's test for variance homogeneity, and presented as mean \pm standard deviation or median (interquartile range). Comparison of ADC, $K_{\text {app }}$, and $D_{\text {app }}$ among different active groups were tested by one-way analysis of variance (ANOVA) or Kruskal-Wallis test. The correlations of between diffusion parameters and SES-CD or MaRIA were tested by spearman rank test. Receiver operating characteristic (ROC) curves were analyzed to assess the ability of diffusion parameters differentiating inactive from active group and inactive-mild from moderate-severe group, and areas under the ROC curves (AUCs) were calculated. The Delong test was used to compare the metrics in term of AUCs. The cutoff value was determined by maximizing the Youden's index. Interobserver consistency of the parameters between the two readers was assessed using the intraclass correlation coefficient (ICC) with 95\% CIs.

\section{Results}

\section{Baseline characteristics of the patients}

A total of 51 patients ( 37 males and 14 females), 127 bowel segments (15 inactive, 45 mild and 67 moderate-severe) were included in the study, including four jejunum, 32 proximal ileum, 43 terminal ileum, 18 cecum/right colon, nine transverse colon, 16 left/sigmoid colon and five rectum segments. The baseline characteristics of the patients were summarized in Table 2.

\section{Correlation between diffusion parameters and SES-CD}

Considering 127 segments, ADC $(r=-0.627, p<0.001)$, $D_{\text {app }}(r=-0.381, p<0.001)$ and $K_{\text {app }}(r=0.641, p<0.001)$ were correlated with SES-CD. ADC $(r=-0.563, p<0.001)$, $D_{\text {app }}(r=-0.306, p<0.001)$ and $K_{\text {app }}(r=0.581, p<0.001)$ were correlated with MaRIA. And segmental MaRIA was correlated with segmental SES-CD $(r=0.741, p<0.001)$.

Regarding jejunum-ileum segments $(n=79)$, ADC $(r=-0.691, p<0.001), D_{\text {app }}(r=-0.409, p<0.001)$ and $K_{\text {app }}(r=0.686, p<0.001)$ were correlated with SES-CD. ADC $(r=-0.631, p<0.001), D_{\text {app }}(r=-0.297, p=0.008)$ and $K_{\text {app }}(r=0.627, p<0.001)$ were correlated with MaRIA. Segmental MaRIA was correlated with segmental SES-CD $(r=0.732, p<0.001)$.

Regarding colorectal segments $(n=48)$, ADC $(r=-0.486, p<0.001), D_{\text {app }}(r=-0.374, p=0.009)$ and $K_{\text {app }}(r=0.510, p<0.001)$ were correlated with SES-CD. ADC $(r=-0.425, p<0.001), D_{\text {app }}(r=-0.338, p=0.019)$ and $K_{\text {app }}(r=0.530, p<0.001)$ were correlated with MaRIA. And segmental MaRIA was correlated with segmental SES$\mathrm{CD}(r=0.755, p<0.001)$.

Table 2 Characteristics of the Crohn's disease patients $(n=51)$

\begin{tabular}{ll}
\hline Male gender $[n(\%)]$ & $37(72.5 \%)$ \\
Age [median (IQR)] & $29(22-40)$ \\
Montreal classification & \\
Disease location $[n(\%)]$ & $19(37.3 \%)$ \\
L1 & $5(9.8 \%)$ \\
L2 & $23(45.1 \%)$ \\
L3 & $4(7.8 \%)$ \\
L4 or L1+L4: & \\
Behavior $[n(\%)]$ & $26(51.0 \%)$ \\
B1 & $14(27.5 \%)$ \\
B2 & $11(21.6 \%)$ \\
B3 & $6(11.8 \%)$ \\
$p$ & \\
Interval (day) & $45(88.2 \%)$ \\
$\leq 7[n(\%)]$ & $6(11.8 \%)$ \\
$7-14[n(\%)]$ & $7.0(5.0-9.0)$ \\
HBI [median (IQR)] & $22.0(12.0-44.0)$ \\
CRP [median (IQR), mg/l] & $23.6(7.2-46.9)$ \\
ESR [median (IQR), mg/l] &
\end{tabular}

$n$ number, $I Q R$ interquartile range, $L 1$ ileal, $L 2$ colon, $L 3$ ileocolon, $L 4$ upper gastrointestinal, $B 1$ inflammatory, $B 2$ structuring, $B 3$ penetrating, $p$ perianal, $H B I$ Harvey-Bradshaw index, $C R P$ C-reactive protein, ESR erythrocyte sedimentation rate 


\section{Differences of the parameters of DKI and DWI among different active groups}

Considering 127 segments, ADC (Kruskal-Wallis test), $D_{\text {app }}$ and $K_{\text {app }}$ (ANOVA) was significantly different (all $p<0.001$ ) among inactive, mild and moderate-severe groups (Fig. 1a-i). The difference of ADC, $D_{\text {app }}$ and $K_{\text {app }}$ among inactive, mild and moderate-severe groups were presented with Fig. 2a-c. When taking into account colorectal segments and jejunum-ileum segments separately, there were still significant difference for ADC, $D_{\text {app }}$ and $K_{\text {app }}$ among different groups (all $p<0.001$ ).

\section{ROC analysis for DKI and DWI to differentiate different active groups (Table 3 )}

Considering 127 segments, ROC analysis found ADC had the highest accuracy ( $\mathrm{AUC}=0.884, p<0.001$ ) to differentiate inactive from active with $93.3 \%$ sensitivity and $77.7 \%$ specificity when ADC was the threshold at $0.865 \times 10^{-3} \mathrm{~mm}^{2} / \mathrm{s}$. The accuracy of $K_{\text {app }}(\mathrm{AUC}=0.867$, $p<0.001$ ) with $67.9 \%$ sensitivity and $93.3 \%$ specificity to differentiate inactive from active was near to ADC with the threshold at 0.645 . However, $\mathrm{D}_{\text {app }}(\mathrm{AUC}=0.726$, $p=0.005$ ) with $93.3 \%$ sensitivity and $55.4 \%$ specificity was obviously inferior to ADC and $K_{\text {app }}$ with the threshold at $1.365 \times 10^{-3} \mathrm{~mm}^{2} / \mathrm{s}$ (Fig. 3a).

Similar accuracy was found between ADC (AUC $=0.846$, $p<0.001$ ) with $71.7 \%$ sensitivity and $89.6 \%$ specificity and $\mathrm{K}_{\mathrm{app}}(\mathrm{AUC}=0.843, p<0.001)$ with $71.6 \%$ sensitivity and $83.3 \%$ specificity to differentiate inactive-mild from moderate-severe at the thresholds of $0.825 \times 10^{-3} \mathrm{~mm}^{2} / \mathrm{s}$ and 0.645 , respectively. $D_{\text {app }}$ (AUC $\left.=0.690, p<0.001\right)$ with $66.7 \%$ sensitivity and $70.1 \%$ specificity was obviously worser than ADC and $K_{\text {app }}$ for differentiating inactive-mild from moderate-severe at the threshold of $1.375 \times 10^{-3} \mathrm{~mm}^{2} / \mathrm{s}$ (Fig. 3b). The Delong test suggested that there were no significant differences for the AUCs of ADC and $K_{\text {app }}$ to differentiate inactive from active $(p=0.895)$ and to differentiate inactive-mild from moderate-severe $(p=0.522)$.

Considering jejunum-ileum segments, the accuracy of $K_{\text {app }}$ (AUC $\left.=0.0 .877, p<0.001\right)$ to differentiating inactive and active was minimally lower than ADC (AUC $=0.891$, $p<0.001)$. Similar results were found in differentiating inactive-mild and moderate-severe for ADC $(\mathrm{AUC}=0.868$, $p<0.001)$ and $K_{\text {app }}(\mathrm{AUC}=0.856, p<0.001)$. However, $D_{\text {app }}$ was obviously lower than ADC and $K_{\text {app }}$ for differentiating inactive from active(AUC $=0.739, p=0.015)$, and for differentiating inactive-mild from moderate-severe (AUC $=0.664, p=0.012$ ). The Delong test suggested that there were no significant difference for the AUCs of ADC and $K_{\text {app }}$ to differentiate inactive from active $(p=0.830)$ and to differentiate inactive-mild from moderate-severe $(p=0.799)$.

Considering colorectal segments, $K_{\text {app }}$ had slightly higher accuracy than ADC to differentiate inactive from active $(\mathrm{AUC}=0.879, p<0.001 \mathrm{vs} . \mathrm{AUC}=0.872, p<0.001)$ and inactive-mild from moderate-severe $(\mathrm{AUC}=0.822$, $p<0.001$ vs. $\mathrm{AUC}=0.801, p<0.001) . D_{\text {app }}$ was unable to differentiate inactive from active $(p=0.147)$, and had significantly lower accuracy than ADC and $K_{\text {app }}$ to differentiate inactive-mild from moderate-severe. The Delong test suggested that there were no significant differences for the AUCs of ADC and $K_{\text {app }}$ to differentiate inactive from active $(p=0.953)$ and to differentiate inactive-mild from moderate-severe $(p=0.774)$.

\section{Interobserver agreement}

The intraclass correlation coefficient were excellent between W.K. and W.G. for ADC [ICC $=0.903$, $(95 \%$ CI 0.891-0.932)] $D_{\text {app }}$ [ICC $=0.897$, (95\% CI 0.882-0.915)], and $\mathrm{K}_{\text {app }}[\mathrm{ICC}=0.911$, (95\% CI 0.901-0.938)].

\section{Discussion}

In our study, it was observed that $\mathrm{ADC}$ and $D_{\text {app }}$ values inversely correlated, and $K_{\text {app }}$ positively correlated with disease activity. The similar AUCs of ADC and $K_{\text {app }}$ were found in differentiating inactive from active and from inactive-mild to moderate-severe; however, the AUCs of $\mathrm{D}_{\text {app }}$ were obviously lower than those of ADC and $K_{\text {app }}$ when analyzing all segments, even jejunum-ileum and colorectal segments, respectively.

The ADC originating from DWI quantitatively reflects water molecule diffusion in tissue in the Gaussian distribution. $D_{\text {app }}$ is true diffusivity, and $K_{\text {app }}$ represents the peaked distribution of tissue diffusivity in the non-Gaussian distribution, which is believed to be associated with microstructural complexity in vivo [10]. With the increasing CD lesion activity, lymphoid tissue, and capillary proliferation, inflammatory cell infiltrations are more prominent, which result in higher restriction of water molecule movement, and manifested as lower ADC. Likewise, these changes coupled with nuclear heterogeneity and cellular complexity result in lower $D_{\text {app }}$ and higher $K_{\text {app }}$. Interestingly, in our study, the AUCs of $D_{\text {app }}$ were obviously lower than those of ADC and $K_{\text {app }}$. Possibly, the complexity in lesion changed more obviously than water molecule diffusion with the increasing CD lesion activity. Actually, ADC calculated in the ideal Gaussian distribution were truly and concisely not able to reflect water molecule diffusion in lesion. Clinically, DKI and DWI could be alternatives to intravenous administration 
Fig. 1 A 39-year-old woman with moderate-severe $\mathrm{CD}$ in the proximal ileum, with SES-CD of 9 and MaRIA of 16.3. Coronal and axial contrast-enhanced $\mathrm{T} 1-(\mathbf{a}, \mathbf{b})$ and $\mathrm{T} 2$-weighted imaging $(\mathbf{c}, \mathbf{d})$ show asymmetric bowel thickening along the mesenteric border (white arrows), sacculations (yellow triangles), and stenosis (white asterisk). Linear hyperintensity (yellow arrow) was found in axial fatsaturated T2-weighted imaging. Hyperintensity present in axial DWI image (e) with b value of $1000 \mathrm{~s} / \mathrm{mm}^{2}$. The endoscopic image of the proximal ileum (f) showed longitudinal ulcer. $\mathrm{ADC}$ map (g), the mean ADC is $0.86 \times 10^{-3} \mathrm{~mm}^{2} / \mathrm{s}$. Diffusivity map (h), the mean $D_{\text {app }}$ was $1.49 \times 10^{-3} \mathrm{~mm}^{2} / \mathrm{s}$. Kurtosis map (i), the mean $K_{\text {app }}$ was 0.65

Fig. 2 ADC, $D_{\text {app}}$, and $K_{\text {app }}$ showing significant differences among inactive, mild, and moderate-severe groups . ADC (a) and $D_{\text {app }}$ (b) decreased with the increasing disease activity, whereas $K_{\text {app }}(\mathbf{c})$ increased (a)

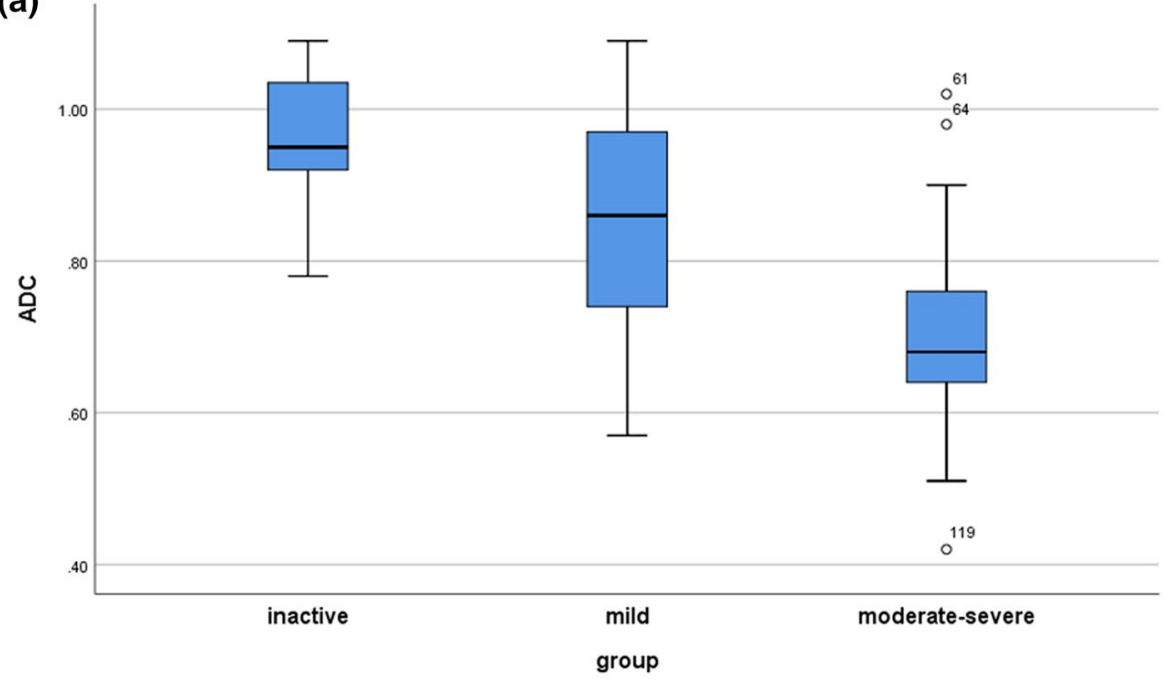

(b)

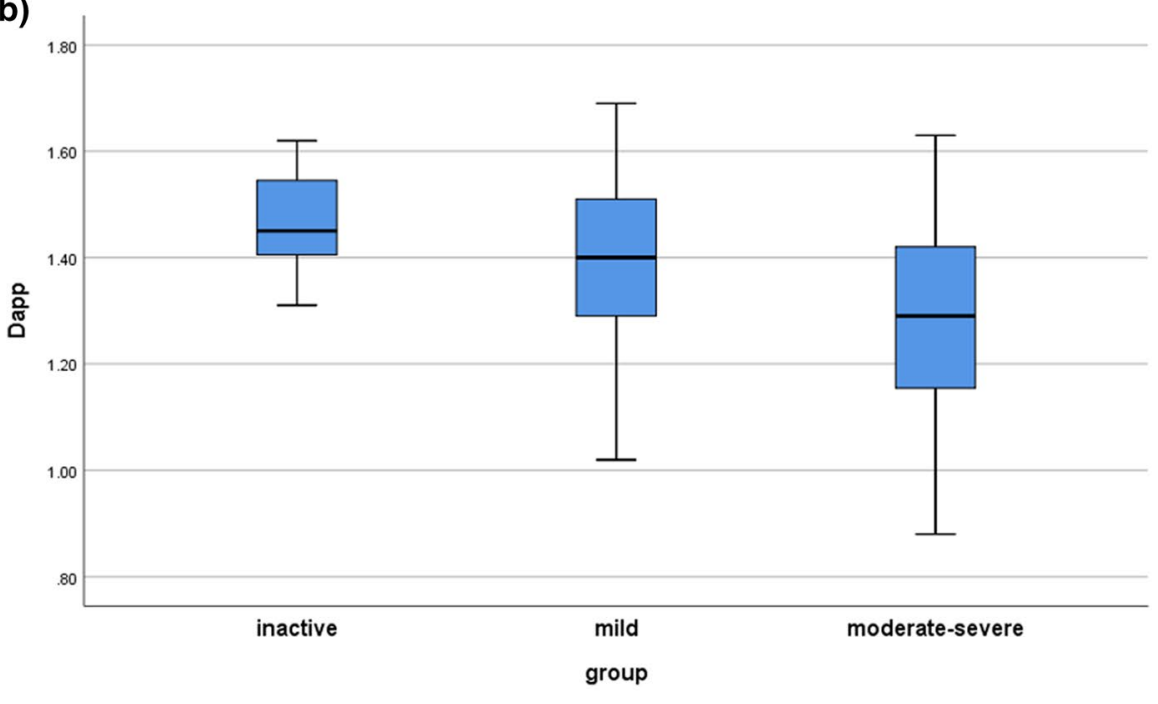

(c)

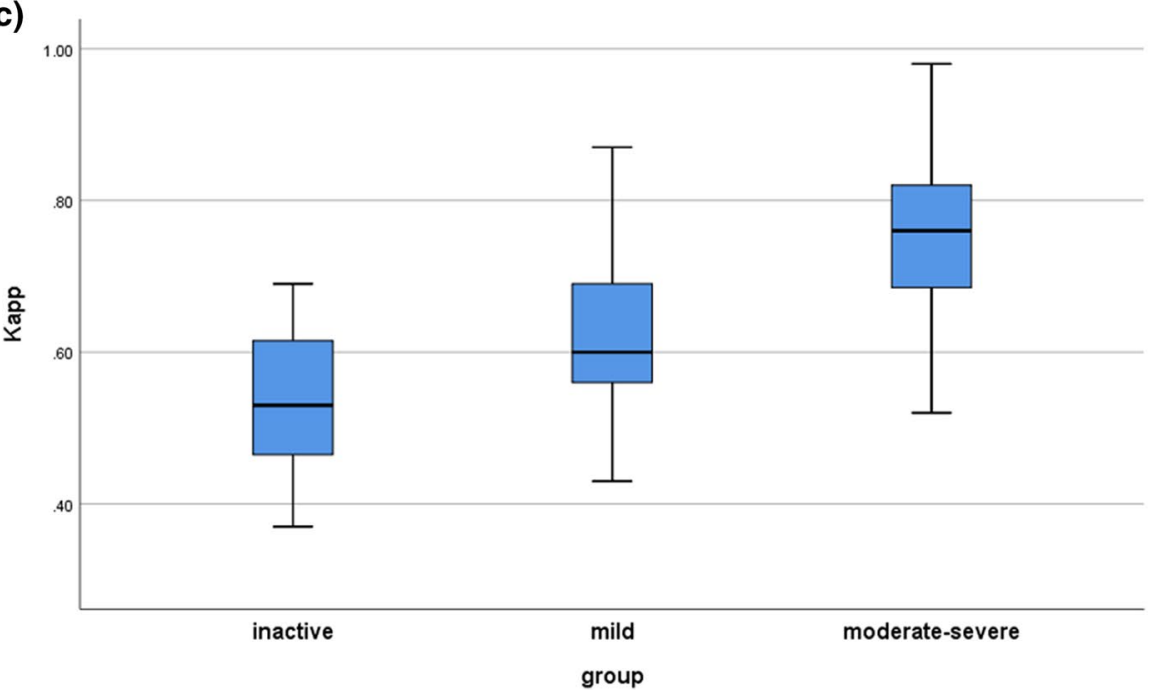


Table 3 ROC analysis for DKI and DWI to differentiate from inactive to active and from inactive-mild to moderatesevere

\begin{tabular}{|c|c|c|c|c|c|c|c|c|c|}
\hline & \multicolumn{3}{|l|}{$\mathrm{ADC}$} & \multicolumn{3}{|l|}{$K_{\text {app }}$} & \multicolumn{3}{|l|}{$D_{\text {app }}$} \\
\hline & AUC & Sen & Spec & AUC & Sen & Spec & AUC & Sen & Spec \\
\hline \multicolumn{10}{|l|}{ Inactive vs. active } \\
\hline All segments & $0.884 * *$ & 0.933 & 0.777 & $0.867 * *$ & 0.679 & 0.933 & $0.726^{*}$ & 0.933 & 0.554 \\
\hline Jejunum-ileum & $0.891 * *$ & 1.00 & 0.812 & $0.877 * *$ & 0.812 & 0.800 & $0.739 *$ & 0.900 & 0.594 \\
\hline Colorectum & $0.872 *$ & 1.00 & 0.651 & $0.879^{*}$ & 0.860 & 0.800 & N.S. & N.S. & N.S. \\
\hline \multicolumn{10}{|c|}{ Inactive-mild vs. moderate-severe } \\
\hline All segments & $0.846^{*}$ & 0.717 & 0.896 & $0.843 * *$ & 0.716 & 0.833 & $0.690 *$ & 0.667 & 0.701 \\
\hline Jejunum-ileum & $0.868 * *$ & 0.750 & 0.872 & $0.856 * *$ & 0.846 & 0.775 & $0.664 *$ & 0.800 & 0.538 \\
\hline Colorectum & $0.801 * *$ & 0.650 & 0.929 & $0.822 * *$ & 0.796 & 0.750 & $0.762 *$ & 0.800 & 0.679 \\
\hline
\end{tabular}

of a gadolinium chelate to assess disease activity. Besides, DKI could provide more useful information about lesion.

Recently, two studies [17, 18] have reported that with DKI it is clinically feasible to evaluate the activity of autoimmune inflammation diseases. Furthermore, Huang et al. [18] found that DKI was able to accurately grade disease activity of $\mathrm{CD}$, even superior to ADC. In our study, the highest accuracy for ADC to differentiate inactive from active was the threshold at $0.865 \times 10^{-3} \mathrm{~mm}^{2} / \mathrm{s}$, and the threshold for $K_{\text {app }}$ was at 0.645 . The thresholds of both ADC and $K_{\text {app }}$ were smaller than that reported in the previous study [19]. Similarly, the trend also occurred in differentiating inactive-mild from moderate-severe. These phenomena were possibly associated with imaging equipment, parameters, and post-processing software we performed in the study. In addition, $K_{\text {app }}$ had similar accuracy with ADC for differentiating inactive from active or inactive-mild from moderate-severe compared to a recent study. The possible reason is that all lesions were divided into groups based on SES-CD rather than MaRIA [18].

Considering the differences between jejunum and ileum and colorectum physiologically, jejunum-ileum and colorectal segments were analyzed separately in this study. We found that the correlations of ADC, $D_{\text {app }}$, and $K_{\text {app }}$ with SES$\mathrm{CD}$ in jejunum-ileum were higher than that in colorectum, but the accuracies to differentiate inactive from active or inactive-mild from moderate-severe in colorectum were similar with that in jejunum-ileum. Therefore, we decided to put jejunum-ileum and colorectum together.

In the study, five $b$ values $(200,500,1000,1500$, and $2000 \mathrm{~mm}^{2} / \mathrm{s}$ ) were applied to acquire DKI imaging. Previous studies $[11,16]$ have demonstrated that perfusion has prominent influence for low $b$ values (usually $<200 \mathrm{~mm}^{2} / \mathrm{s}$ ); nonetheless, too much high $b$ values increase the chance of image distortion and susceptibility artifacts, especially for bowels. Therefore, considering acquisition time, we finally selected above five $b$ values in our institution.
Regarding ROI placement on DKI, each ROI was manually drawn along the border of the brightest signal region of bowel wall, instead of size-fixed (i.e. $6-8 \mathrm{~mm}^{2}$ ) round ROIs [15]. We suggested that there might be selection bias placing small size-fixed round ROIs. A recent study [20] suggested that to analyze a larger number of pixels may result in more reproducible results for the measured parameters. Furthermore, We chose consecutive three slices of ROIs (the slice with the prominent lesion section and the adjacent up and down slices), thus could decrease the deviation of sampling between cases and increase the reliability of results [16].

There are some limitations in our study. Firstly, this is retrospective study, meaning that there were potential selection biases. Secondly, the number of bowel segments was relatively less, because the number of patients was insufficient and normal segments were excluded. Besides, the number of inactive segments was significantly less than mild or moderate-severe, because most of inactive lesions and partial mild lesions, such as erosion, erythema, edema, small ulcers, can be detected by endoscopy, however, they were hardly displayed on conventional MR images, especially on high $b$ values images resulting from the decrease of the signal-to-noise ratio. So our results were not fully and concisely able to reflect the difference of diffusion parameters among different active lesions. Thirdly, due to the decrease of the signal-to-noise ratio on high $b$ values and thin bowel wall, drawing outline ROI inevitably resulted in containing intestinal contents. Finally, although we found DKI was comparable to conventional DWI to assess disease activity in our study, the feasibility of DKI on evaluating disease activity in $\mathrm{CD}$ need to be studied through large prospective multicenter studies.

In conclusion, this study suggested that DKI is clinically feasible and helpful to evaluate disease activity in CD. DKI is comparable to conventional DWI for grading disease activity, and able to provide more useful information about lesion. 


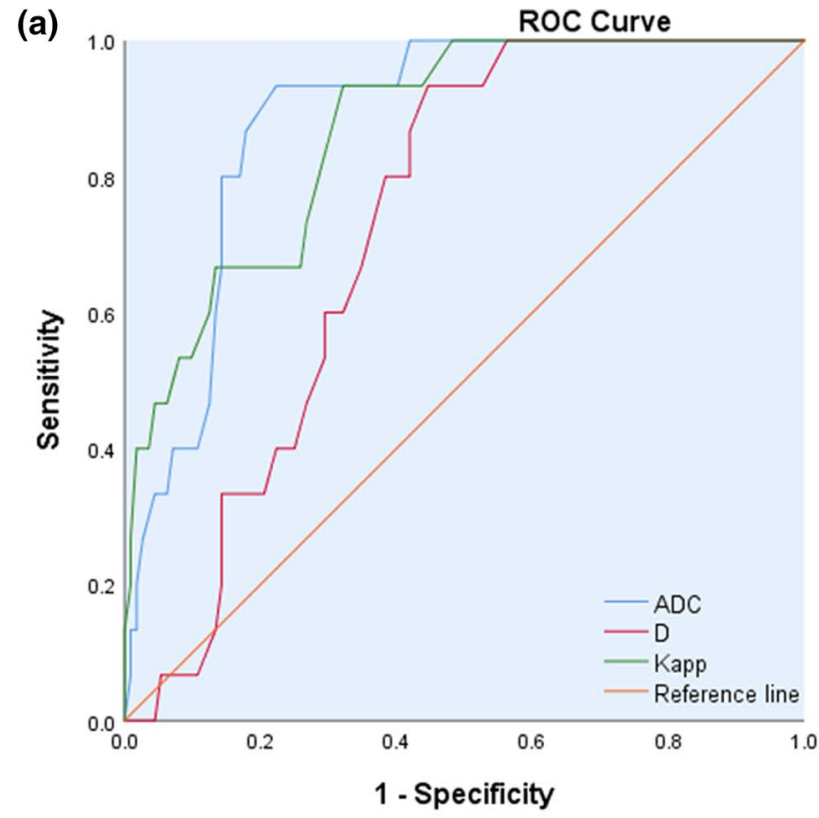

(b)

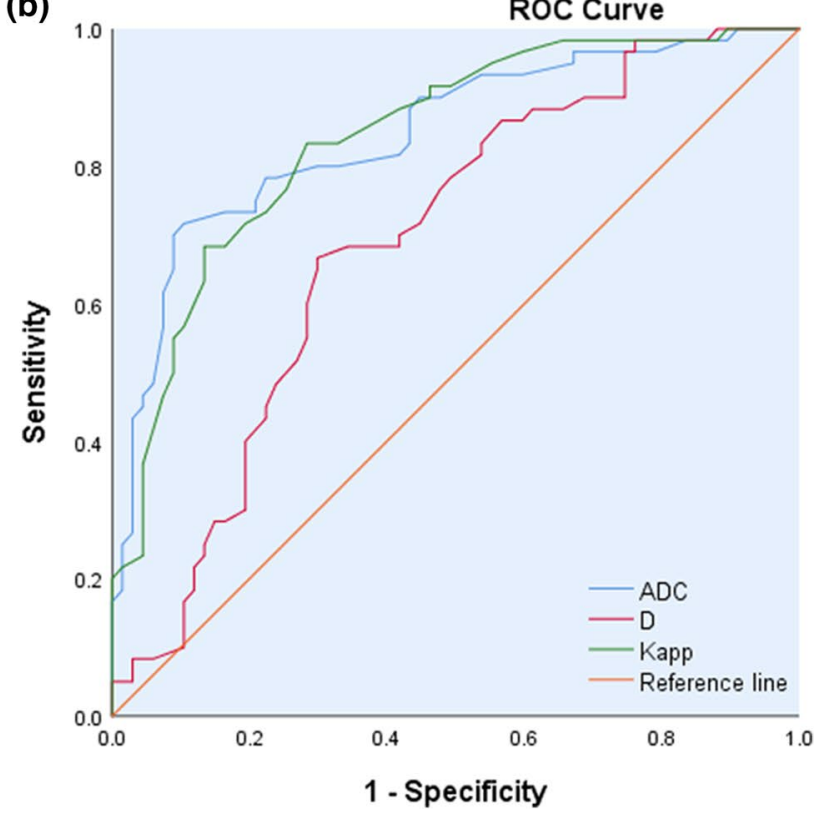

Fig. 3 ROC analysis considering 127 bowel segments; ADC was found to have the highest accuracy ( $\mathrm{AUC}=0.884, p<0.001$ ) to differentiate inactive from active group, which was slightly higher than $K_{\text {app }}(\mathrm{AUC}=0.867, p<0.001)$, and obviously higher than $D_{\text {app }}$ (AUC $=0.726, p=0.005)$ (a). For differentiating inactive-mild from moderate-severe group (b), ADC also had the highest accuracy $(\mathrm{AUC}=0.846, p<0.001$ ), which was minimally higher than $K_{\text {app }}(\mathrm{AUC}=0.843, p<0.001)$, and obviously higher than $D_{\text {app }}$ (AUC $=0.690, p<0.001)$

Acknowledgements This study was supported by the National Natural Science Foundation of China (Grant No. 81227902), the National Natural Science Foundation of China (Grant No. 2016YFC1304702) and Shenzhen University Presidential Fund 85706-0000040544.
Data availability The data that support the findings of this study are available on request from the corresponding author (Guangyao $\mathrm{Wu}$ ). The data are not publicly available because the aforementioned data containing information that could compromise research participant privacy.

\section{Compliance with ethical standards}

Conflict of interest The authors declare that they have no conflict of interest.

Open Access This article is distributed under the terms of the Creative Commons Attribution 4.0 International License (http://creativeco mmons.org/licenses/by/4.0/), which permits unrestricted use, distribution, and reproduction in any medium, provided you give appropriate credit to the original author(s) and the source, provide a link to the Creative Commons license, and indicate if changes were made.

\section{References}

1. Torres J, Mehandru S, Colombel JF, et al. (2017) Crohn's disease. LANCET 389:1741-1755.

2. Ordas I, Rimola J, Rodriguez S, et al. (2014) Accuracy of magnetic resonance enterography in assessing response to therapy and mucosal healing in patients with Crohn's disease. GASTROENTEROLOGY 146:374-382.

3. Fernandes SR, Rodrigues RV, Bernardo S, et al. (2017) Transmural Healing Is Associated with Improved Long-term Outcomes of Patients with Crohn's Disease. INFLAMM BOWEL DIS 23:1403-1409.

4. Takenaka K, Ohtsuka K, Kitazume Y, et al. (2018) Utility of Magnetic Resonance Enterography For Small Bowel Endoscopic Healing in Patients With Crohn's Disease. AM J GASTROENTEROL 113:283-294.

5. Coimbra AJ, Rimola J, O'Byrne S, et al. (2016) Magnetic resonance enterography is feasible and reliable in multicenter clinical trials in patients with Crohn's disease, and may help select subjects with active inflammation. Aliment Pharmacol Ther 43:61-72.

6. Rimola J, Ordas I, Rodriguez S, et al. (2011) Magnetic resonance imaging for evaluation of Crohn's disease: validation of parameters of severity and quantitative index of activity. INFLAMM BOWEL DIS 17:1759-1768.

7. Barat M, Hoeffel C, Bouquot M, et al. (2019) Preoperative evaluation of small bowel complications in Crohn's disease: comparison of diffusion-weighted and contrast-enhanced MR imaging. EUR RADIOL 29:2034-2044.

8. Lanier MH, Shetty AS, Salter A, et al. (2018) Evaluation of noncontrast MR enterography for pediatric inflammatory bowel disease assessment. J MAGN RESON IMAGING 48:341-348.

9. Qi F, Jun S, Qi QY, et al. (2015) Utility of the diffusion-weighted imaging for activity evaluation in Crohn's disease patients underwent magnetic resonance enterography. BMC GASTROENTEROL 15:12.

10. Jensen JH, Helpern JA, Ramani A, et al. (2005) Diffusional kurtosis imaging: the quantification of non-gaussian water diffusion by means of magnetic resonance imaging. MAGN RESON MED 53:1432-1440.

11. Wan Q, Deng YS, Lei Q, et al. (2019) Differentiating between malignant and benign solid solitary pulmonary lesions: are intravoxel incoherent motion and diffusion kurtosis imaging superior 
to conventional diffusion-weighted imaging? EUR RADIOL 29:1607-1615.

12. Huang Y, Lin Y, Hu W, et al. (2019) Diffusion Kurtosis at 3.0T as an in vivo Imaging Marker for Breast Cancer Characterization: Correlation With Prognostic Factors. J MAGN RESON IMAGING 49:845-856.

13. Xie H, Wu G (2018) Application of Diffusion Kurtosis Imaging and Histogram Analysis for Assessing Preoperative Stages of Rectal Cancer. Gastroenterol Res Pract 2018:9786932.

14. Mary JY, Modigliani R (1989) Development and validation of an endoscopic index of the severity for Crohn's disease: a prospective multicentre study. Groupe d'Etudes Therapeutiques des Affections Inflammatoires du Tube Digestif (GETAID). GUT 30:983-989.

15. Radmard AR, Eftekhar VR, Montazeri SA, et al. (2018) Mesenteric lymph nodes in MR enterography: are they reliable followers of bowel in active Crohn's disease? EUR RADIOL 28:4429-4437.

16. Wang X, Tu N, Qin T, et al. (2018) Diffusion Kurtosis Imaging Combined With DWI at 3-T MRI for Detection and Assessment of Aggressiveness of Prostate Cancer. AJR Am J Roentgenol 211:797-804.

17. Wang F, Chu C, Zhao C, et al. (2019) Diffusion kurtosis imaging in sacroiliitis to evaluate the activity of ankylosing spondylitis. $\mathbf{J}$ MAGN RESON IMAGING 49:101-108.
18. Huang L, Li XH, Huang SY, et al. (2018) Diffusion kurtosis MRI versus conventional diffusion-weighted imaging for evaluating inflammatory activity in Crohn's disease. J MAGN RESON IMAGING 47:702-709.

19. Buisson A, Joubert A, Montoriol PF, et al. (2013) Diffusionweighted magnetic resonance imaging for detecting and assessing ileal inflammation in Crohn's disease. Aliment Pharmacol Ther 37:537-545.

20. Sun Y, Xiao Q, Hu F, et al. (2018) Diffusion kurtosis imaging in the characterisation of rectal cancer: utilizing the most repeatable region-of-interest strategy for diffusion parameters on a 3T scanner. EUR RADIOL 28:5211-5220.

Publisher's Note Springer Nature remains neutral with regard to jurisdictional claims in published maps and institutional affiliations. 